REVUE GÉOGRAPHIQUE DES PYRÉNÉES ET DU SUD-OUEST TOME 59, FASC. 1 , pp. 73-88, Toulouse, 1988.

\title{
Un torrent oublié \\ mais catastrophique en Haute-Ariège
}

\author{
par Jean.Marc ANTOINE *
}

Nous connaissons bien la crise érosive de la fin du $\mathrm{XIX}^{\mathrm{e}}$ siècle dans les Pyrénées : ouragans, éboulements, laves torrentielles, inondations, qui sont à l'origine de l'attention apportée par l'administration forestière à la question de la torrentialité; de là viennent les lois de 1860,1864 et surtout 1882 , cette dernière visant la restauration des terrains en montagne. L'occurrence de cette crise ne prête pas à discussion. Mais les avis divergent sur ses causes. Les forestiers du siècle dernier y ont vu la conséquence inévitable des déboisements intensifs, des " extirpations " du XVII", du XVIII et même du $\mathrm{XIX}^{\circ}$ siècle et celle de l'augmentation de la pression pastorale, liée, entre autres, à la croissance démographique des populations pyrénéennes du milieu du $\mathrm{XIX}^{\cdot}$ siècle. Nous nous demandons aujourd'hui si cette crise n'était pas liée à une péjoration climatique, ce qui induit la notion de crise catastrophique; la vérification de cette hypothèse réclame une meilleure connaissance de cette période et du contexte historique des événements : dénombrement et chronologie des catastrophes repérables à partir du XVII ${ }^{-}$siècle et recherche de leurs causes possibles.

C'est ce que nous avons tenté dans le val d'Ariège, - la haute vallée de l'Ariège, entre Tarascon et Ax-les-Thermes. Nous avons pu y distinguer des cycles catastrophiques vraisemblables d'abord grâce à l'examen des sources d'archives et des sources bibliographiques, ensuite et avec les connaissances recueillies de la sorte grâce à l'analyse sur le terrain des cônes de déjection. La trame historique et chronologique esquissée grâce aux documents écrits pouvait alors être

(*) Etudiant en doctorat de géographie, Université de Toulouse-Le Mirail, 31058 Toulouse Cedex. 
confrontée à la lecture des séquences sédimentaires des cônes, dont nous admettons qu'elles traduisent d'une façon précise l'évolution géomorphologique des bassins-versants.

L'exemple choisi est celui du torrent du Lagal, au Castelet, commune de Perles-Le-Castelet; ce modeste tributaire de rive gauche de l'Ariège, en aval d'Ax-les-Thermes, paraît typique : son bassin, développé en bordure du massif gneissique de l'Aston, illustre bien la sensibilité chronique de bien des milieux montagnards aux risques d'une morphogenèse catastrophique. Bon nombre des facteurs ici répertoriés sont parmi les constantes de la chaîne pyrénéenne; d'autres sont propres à ce petit bassin.

\section{Des traits communs pyrénéens, une originalité locale.}

Comme dans le reste de la montagne, l'épaisseur des dépots d'origine glaciaire et la raideur des pentes, - si l'on considère les héritages -, l'action anthropique et les aléas climatiques, - si l'on examine les éléments conjoncturels -, sont les principaux facteurs d'instabilité et de fragilité.

L'érosion glaciaire a peu joué, mais les accumulations liées aux glaciers emplâtrent cependant les versants jusque vers $1700-1800 \mathrm{~m}$, soit l'altitude maximale atteinte ici par le glacier de l'Ariège. Moraines et dépôts fluvio-glaciaires sont suspendus de part et d'autre du ruisseau du Lagal en position instable, d'autant que les pentes sont fortes : la déclivité est rarement inférieure à $18^{\circ}$, mais est supérieure à $27^{\circ}$ dans le secteur de la gorge de raccordement et elle dépasse parfois $45^{\circ}$ dans la partie amont du bassin-versant; seuls les interfluves, rabotés par le passage du glacier, présentent des pentes relativement faibles. Cette disposition entraîne deux conséquences : l'appel au vide est une constante de ce petit bassin-versant court perché audessus de l'auge de l'Ariège; des abats d'eau importants, comme il en survient souvent dans la région, suffisent dès lors à mobiliser des milliers de mètres cubes de matériel meuble dont les seuils de plasticité et de fluidité ont été atteints. Des orages violents et localisés, mais aussi bien des épisodes pluvieux généralisés et prolongés sont les facteurs déterminants du déclenchement même de tels phénomènes, indépendamment de l'incidence d'autres facteurs conjoncturels, telle la surexploitation des sols et de leur couverture végétale que l'on a trop souvent et exclusivement mise en cause (fig. 1).

La tentation est grande, en effet, d'attribuer l'origine de la crise érosive constatée à la fin du XIX siècle aux conséquences des activités anthropiques et à leurs effets sur le couvert végétal. Mais il faut examiner une telle explication avec prudence. En 1875 le recouvrement végétal était certainement loin de l'optimum. Mais que le taux de boisement et de couverture soit autrement plus élevé aujourd'hui n'a pas contrarié le déclenchement de la lave torrentielle qui a 


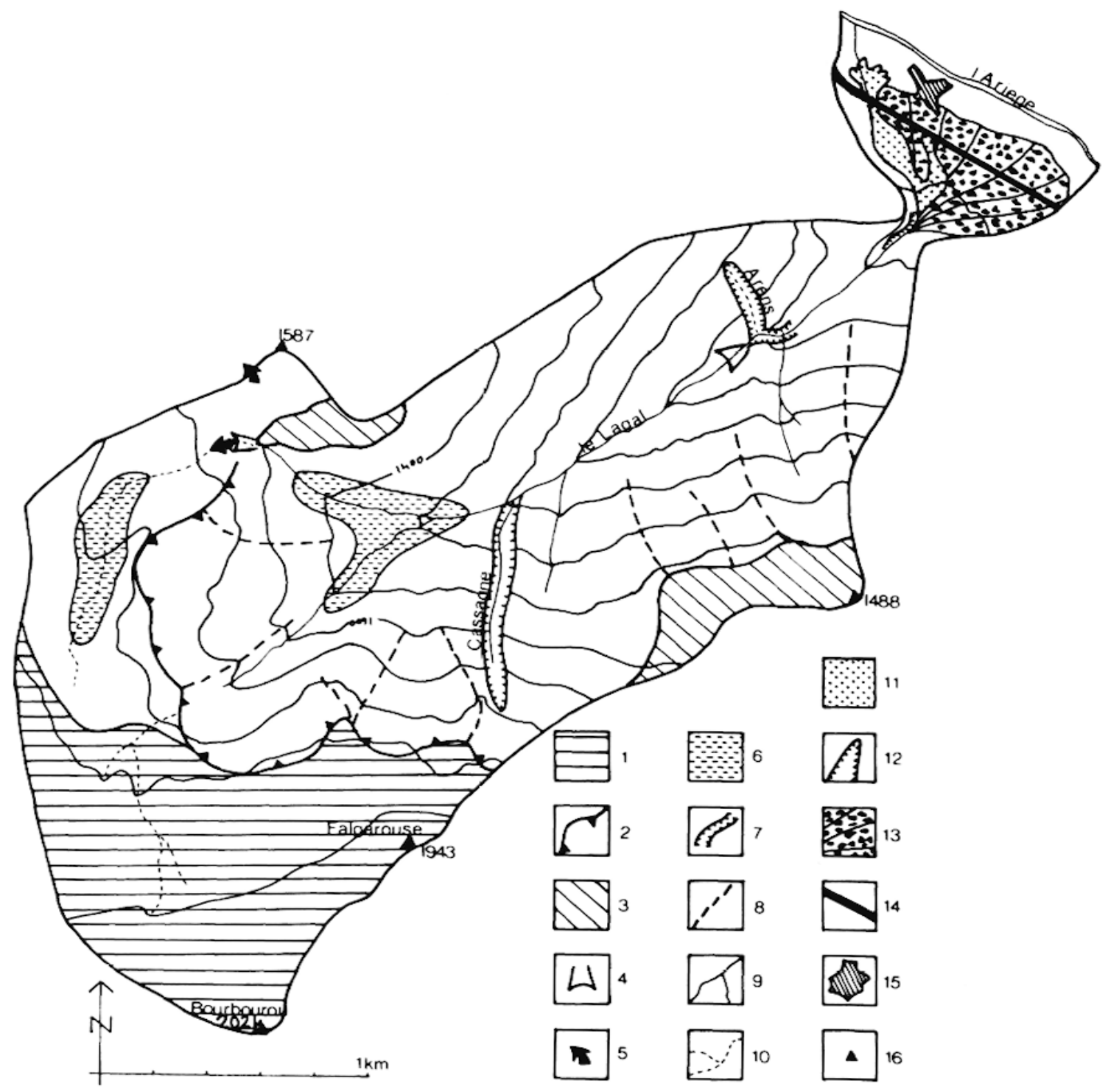

Fig. 1

Croquis gémorphologique du bassin du Lagal.

1. Versant préglaciaire subhorizontal. - 2. Limite de surcreusement glaciaire. 3. Surface plane d'origine glaciaire. - 4. Vallée suspendue. - 5. Difluence, transfluence. - 6. Accumulation de dépôts glaciaires. - 7. Gorge. - 8. Interfluve large. - 9. Chenal incisé avec rives boisées. - 10. Chenal incisé avec rives à vif. - 11. Déjections de la lave de 1986. - 12. Zones d'alimentation des laves de $1772,1875,1881,1910$. - 13. Cône de déjection. - 14. Route nationale 20 . 15. Village. -16 . Points cotés.

déferlé sur le cône de déjection du Castelet au mois de mai 1986. On a cependant souvent mis en relation l'intensification de l'utilisation de l'espace, associée au maximum démographique atteint vers 1860 en Ariège, avec la réactivation d'une torrentialité catastrophique. Tel 
arrêté municipal de 1856 témoigne ainsi au Castelet de la pression alors exercée sur la forêt : le garde champêtre y est autorisé à dresser procés-verbal à qui irait chercher du bois dans la forêt communale sans autorisation (1). On a de même évoqué les conséquences qu'ont pu avoir sur la densité du couvert forestier et la forge du Castelet, signalée dès 1772 (2) et les six forges dénombrées pour le consulat d'Ax sous la "réformation » de Froidour (1668-1673). Nous repérons encore sur les versants de nombreux replats aménagés, à une époque indéterminée, pour des charbonnières servant à approvisionner ces " moulis de fer". Que la surexploitation des étages forestier et supraforestier fût réelle ne suffit pas à lui faire attribuer la responsabilité exclusive de la crise érosive du XIX' siècle; l'abandon récent n'a d'ailleurs pas déterminé une inversion durable de cette dynamique torrentielle.

Sur place, certaines conditions spécifiques ont certainement joué un rôle décisif. La raideur des pentes paraît, dans le cas du Lagal, un élément déterminant de la torrentialité : la valeur de la pente y est en moyenne de $13^{\circ}$ pour une longueur qui n'excède pas $3300 \mathrm{~m}$ à l'apex du cône de déjection; ce chenal principal très court et très pentu s'inscrit d'ailleurs dans un système de versants raides, ce qui amplifie l'effet des processus morphologiques. D'autre part, le fait que le vallon du Lagal soit, du SSW au NNE, perpendiculaire au val d'Ariège (SSE-NNW) peut avoir une certaine incidence : on lui doit l'exposition aux flux atmosphériques responsables des plus gros abats d'eau : la rive gauche est directement soumise aux ascendances liées à la circulation d'WNW. Quelques faits relevés sur le terrain consolident cette hypothèse : en 1875, l'échancrure qui a nourri la lave torrentielle de cette année est apparue en rive droite, par un temps régi par un flux d'ouest; en revanche, la ravine d'Arrens, en rive gauche, semble consécutive aux pluies torrentielles de septembre 1772, lesquelles, comme en témoignent les archives semblent liées à un flux de sud: "dans la nuit du 16 et la journée du 17 septembre, un vent du midi d'une violence épouvantable qui avait été précédé d'une pluie si abondante et si continue " (3). Troisième élément relevant de la configuration locale: la situation du bassin du Lagal sur la marge du plateau de l'Aston où les ascendances orographiques sont très fortes et les orages fréquents.

Le risque d'événements catastrophiques, latent dans la montagne pyrénéenne, est sans doute aggravé par ces dispositions locales particulières. L'examen des sources d'archives confirme d'ailleurs que la fréquence de la torrentialité est un caractère durable du secteur considéré.

(1) Archives départementales de l'Ariège (désormais AD 09), 7 M 2.

(2) AD 09, 1 C 165.

(3) $\mathrm{AD} 09,1 \mathrm{C} 31$. 


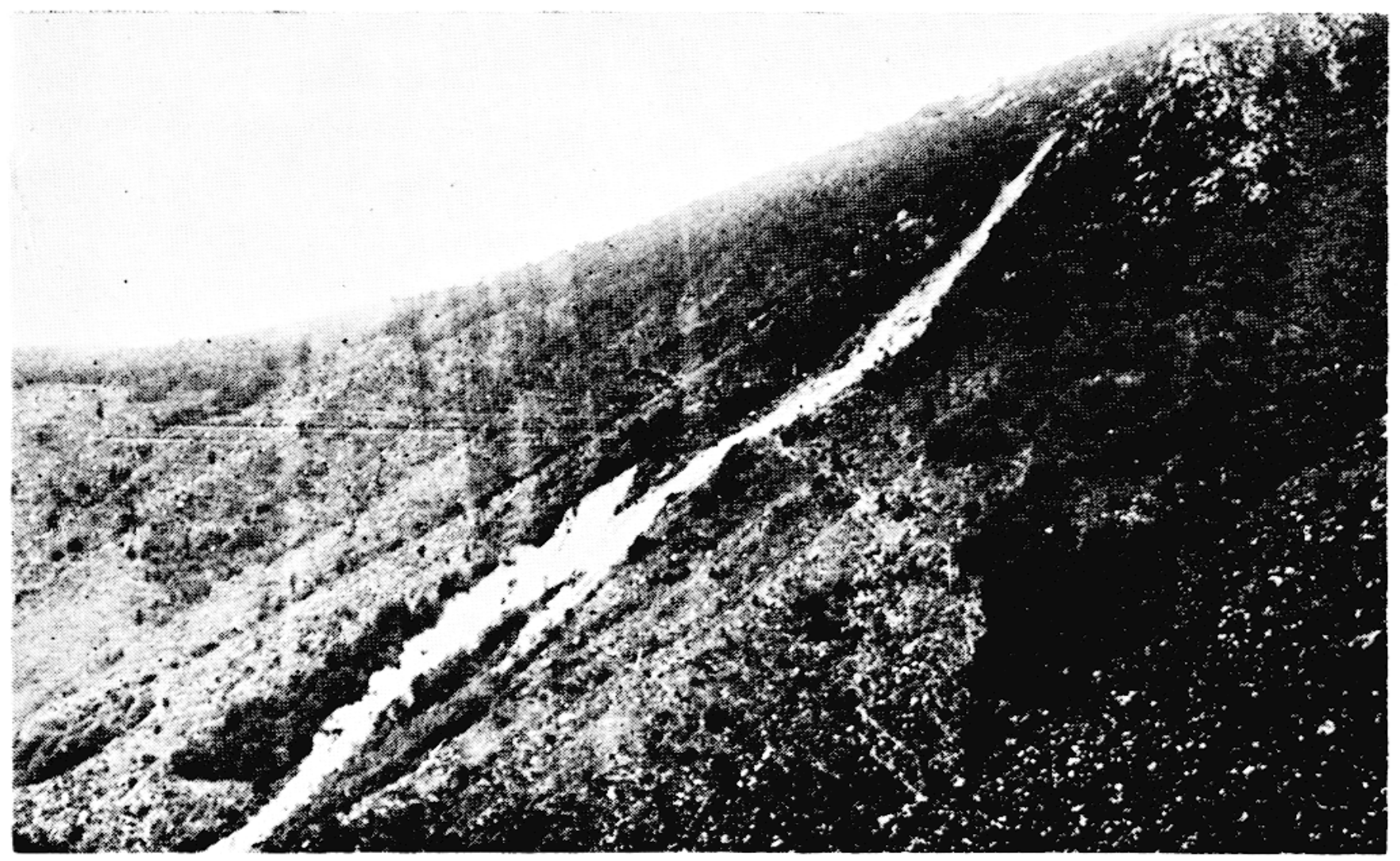

Рното 1

Perles-le-Castelet, 1910

Vue de la partie supérieure du bassin du Lagal, avec la trace des coulées boueuses de 1875 et 1910 . La zone de départ se situe dans les rochers, vers $1650 \mathrm{~m}$; le versant montré a $350 \mathrm{~m}$ de dénivelée. (Cliché Rochebrune).

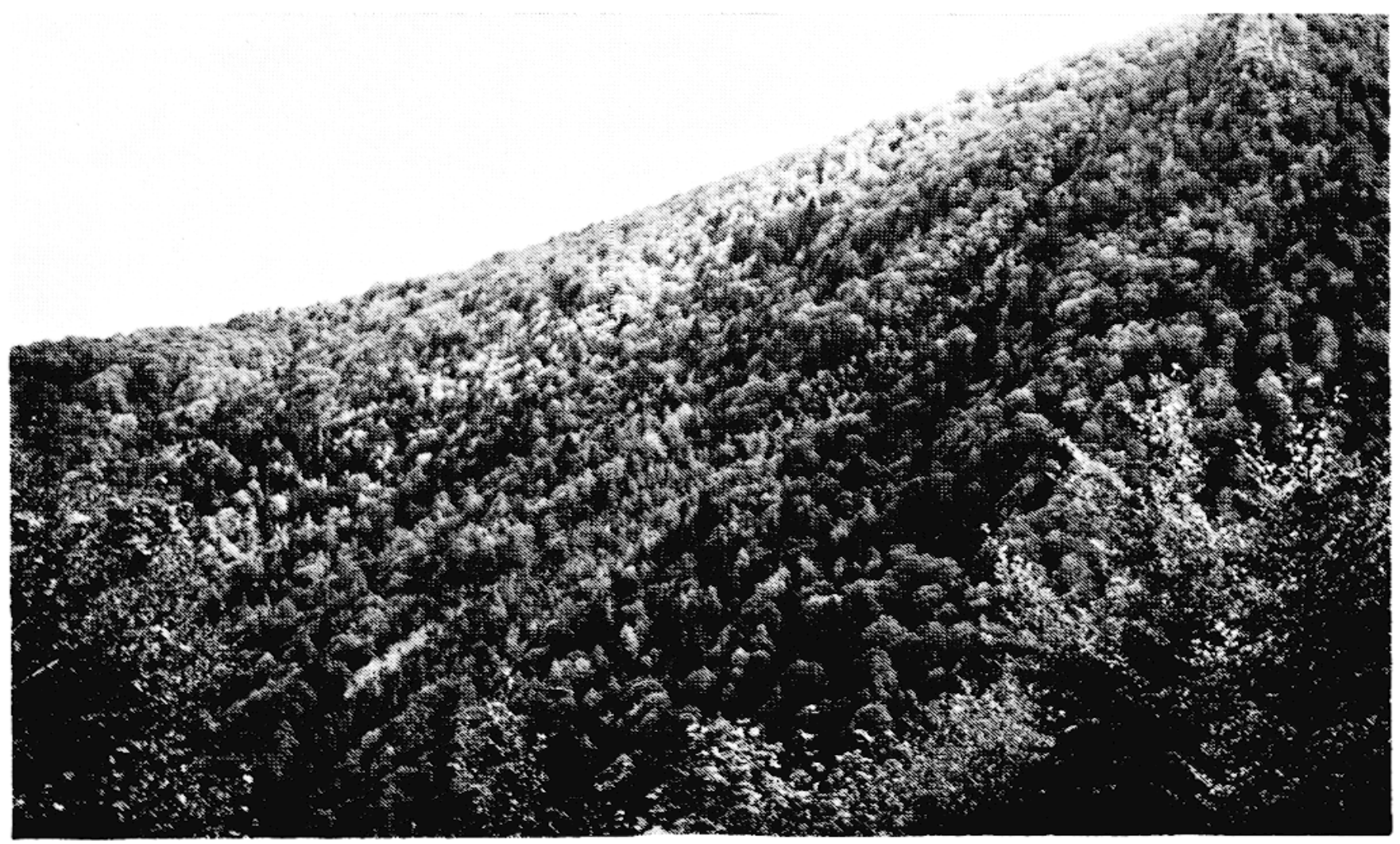

Рното 2

Perles-le-Castelet, 1987

Le versant, autrefois charbonné en totalité, est aujourd'hui envahi par les hêtres, bouleaux et sapins. (Cliché Métailié). 


\section{Fréquence des crises érosives dans la période moderne et contemporaine.}

Il semble bien établi que la distribution des inondations et des laves torrentielles n'est pas aléatoire : le cas du Lagal, considéré depuis le XVIII' siècle, n'est pas séparable de celui de l'ensemble des tributaires de l'Ariège. La période 1850-1910 paraît bien caractérisée par une fréquence paroxystique de la torrentialité.

\section{Deux cycles établis de torrentialité catastrophique.}

Les épisodes de torrentialité catastrophique intéressant le Lagal corespondent à la chronologie établie pour l'ensemble du val d'Ariège, sauf pour ce qui est du XVII' siècle, pendant lequel, faute d'autres preuves, il paraît être resté calme (fig. 2).

Le troisième quart du XVIII siècle fut marquée par une recrudescence de l'activité torrentielle dont le point de départ fut, comme dans le reste du secteur, l'inondation de fin juillet-début août 1750 . Le pont de Perles-et-Castelet fut alors endommagé, des récoltes et des champs emportés dans les parages d'Ax (4).

Vingt ans plus tard, le 20 mai 1770 le ruisseau de Guissou dévasta les champs de la Plaine au Castelet alors que la digue du moulin était emportée par l'Ariège (5). Ni dans l'un, ni dans l'autre cas, le ruisseau de Lagal n'est précisément en cause, mais il contribua vraisemblablement à chacune de ces grandes crues. Nul doute, en revanche, sur son rôle au cours de l'épisode suivant, particulièrement dévastateur : le 17 septembre 1772, une lave torrentielle ravage et engrave les prairies établies sur le cône de déjection aux lieux dits Fouzet, La Gailh, La Mouillère, La Font, La Bestiaque, La Prade (6). Le désastre fut tel qu'on en conserva le souvenir : en 1887, on évoque dans le Projet de périmètre de restauration de la Haute-Ariège "une grande débâcle survenue il y a une centaine d'années et qui est encore dans les souvenirs »(7).

Sans doute les archives ne gardent-elles pas la trace directe des météores de moindre importance ni de leurs conséquences hydrologiques quand elles eurent moins d'importance qu'en 1750,1770 ou 1772. Mais on sait que la haute Ariège a été affectée par un violent orage le 30 août 1762 et qu'en 1767, 1768, 1769, les habitants du pays de Foix et du Roussillon ont eu droit à des allègements sur la capitation " suite aux grêles, inondations et autres cas fortuits " (fig. 2). Admet-

(4) AD 09, 1 C 27.

(5) AD 09, 1 C 30.

(6) AD 09, 1 C 31 .

(7) Archives régionales de Midi-Pyrénécs (dćsormais ARMP). Fonds Office national des Forêt : $3530 / 13$. 


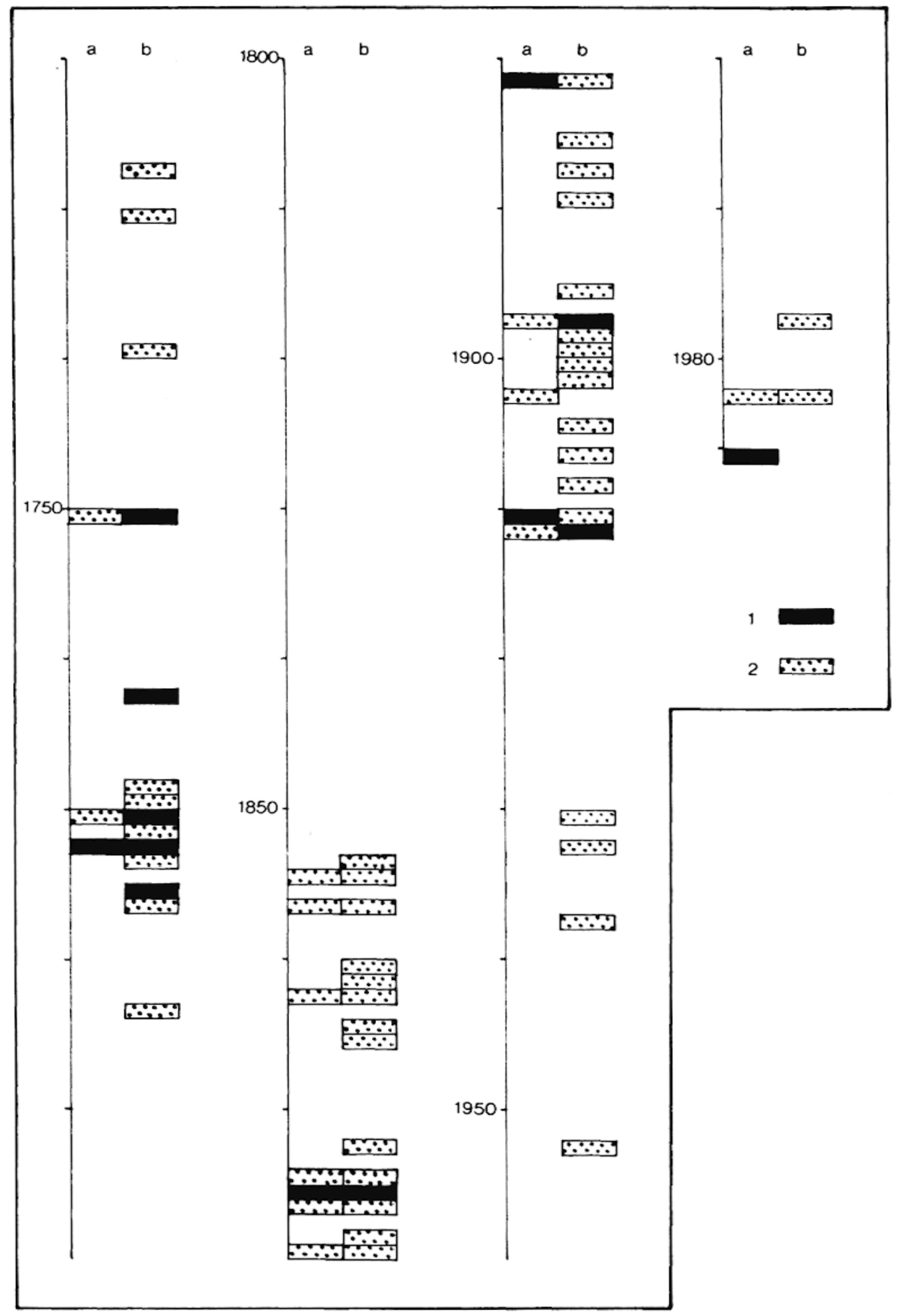

FIG. 2

Chronologie des laves et inondations au Castelet ot on Val d'Ariége du XVIII' au XX⿳ slecle.

1. Laves torrentielles. - 2. Inondations. - a. Site du Castelet. - b. Autres sites en Val d'Ariège. 
tons que cela suffise, pour le moment, à tenir l'ensemble de cette période pour catastrophique (8).

La période 1850-1910 est connue avec plus de précision et nous savons qu'entre 1854 et 1921 douze inondations ont affecté le site du Castelet. Les premières se suivent de près à partir du 12 juin 1874, les 22 et 23 juin 1875, en mai 1876, le 22 décembre 1879, le 16 juin 1881; les secondes environ vingt-cinq à trente ans plus tard : 2 et 3 octobre 1897, 28 juin 1902, 23 mai 1910, mai et juin 1911. Les inondations de 1875,1881 et 1910 furent marquées par d'abondantes vidanges de matériel, dont les deux plus importantes ont entraîné des milliers de mètres cubes de terrains meubles encombrés de rochers et d'arbres. Nous dispcsons pour le désastre de 1875 d'un témoignage révélateur : « Il y eut une pluie torrentielle de 53 heures à partir du 20 juin. Le 23, à 9 heures du matin, une masse très considérable de terre végétale et de sables détrempés par les eaux se détacha avec un épouvantable fracas répercuté par toute la vallée de l'Ariège, jusqu'à Ax, dans le vallon du Lagal, au lieu dit Roucatel-Pounchut, dans le quartier de Falgarouse, et, entraînant sur son passage rochers, arbres, etc., vint barrer ledit et étroit vallon du Planel de la Llabère situé à $1050 \mathrm{~m}$ d'altitude. Vers 11 h 45, son ruisseau, démesurément grossi, accumula ses eaux et finit par rompre cette digue momentanée. En quelques instants, 17 hectares de prairies et de champs furent couverts de blocs, de pierres et de sables »(9). L'épaisseur moyenne de la couche "d'immenses rochers, pierres et sables qui recouvrent champs et prairies au Castelet est de $2 \mathrm{~m}$ et même plus, par endroit »(10). Le flot boueux a d'ailleurs ensablé quelques maisons et granges desquelles on a évacué les vaches avec des palans, "car elles étaient envasées jusqu'au ventre »(11). L'ensemble des dégats fut estimé à 102660 francs (12).

L'éboulement de la combe de Cassagne, qui alimenta la lave de 1875, participa aussi, à la lave de 1881, de même que l'éboulement de la combe d'Arreus dont la première manifestation paraît remonter au plus tard à 1772. Si la vidange de 1881 est la moins importante des trois qui nous retiennent, elle a cependant emporté des digues avant d'engraver des récoltes et d'envahir quelques maisons. C'est probablement un phénomène du même ordre qui s'est produit en 1986 lorsque les atterrissements se sont répandus une fois encore sur le cône, mais sans atteindre le village.

Avec 70000 mètres cubes de matériaux mobilisés, l'ampleur de l'épandage de 1910 évoque beaucoup plus le désastre de 1875 : la

(8) AD 09, 1 C 30.

(9) Témoignage de M. l'abbé Durand cité par H. Marcailhou D'Aymeric, Notice sur les villages composant le canton d'Ax, Foix, Pommiès, 1909, p. 82.

(10) AD 09,7 M 11 .

(11) Témoignage récent d'un pâtre du Castelet qui tenait ce récit de son propre grand-père.

(12) ARMP, Fonds ONF, 3530/18. 
ravine de Cassagne fut à nouveau élargie et recreusée, alors que le village lui-même ne fut pas atteint (photo 1); mais la lave se déversa pendant trente minutes sur une douzaine d'hectares cultivés (13).

Quelles seraient aujourd'hui les conséquences de ces catastrophes importantes et souvent répétées ? Ni les habitants ni le village du Castelet ne paraissent à première vue directement menacés : souvent atteint, le village ne l'a jamais été gravement depuis le XVIII siècle, mais rien ne prouve qu'il soit réellement hors d'atteinte. En revanche, il serait clair que la $\mathrm{RN} 20$ subirait des dégats assez graves pour interrompre durablement le trafic routier; le tracé primitif de cette route passe d'ailleurs sur l'autre rive de l'Ariège, à l'abri des débordements et des atterrissements du Lagal, ce qui témoigne de l'importance qu'avait vraiment le fonctionnement du ruisseau dans la vie quotidienne, ou de celle qu'on lui accordait.

Le troisième quart du $\mathrm{XVIII}^{\bullet}$ siècle et la fin du XIX $\mathrm{X}^{\bullet}$ constituent donc deux périodes de torrentialité active qui se démarquent d'autres années beaucoup plus calmes du reste des deux siècles considérés. Il faudra examiner dans ce contexte chronologique la signification de la lave survenue en mai 1986 et à laquelle on doit plusieurs coupes stratigraphiques fraîches mises à jour dans l'épaisseur du cône.

\section{Stratigraphie du cône de déjection, rythmes de la torrentialité.}

L'examen des coupes stratigraphiques ouvertes par l'épisode torrentiel permet d'envisager la rythmicité des crises érosives attestées par des arguments historiques.

Au Castelet, comme ailleurs dans le val d'Ariège, à Bouan, à Verdun, à Gargantc, à Albiès, à Lassur, etc., les cônes de déjection présentent une alternance de lits sablo-limoneux et de lits caillouteux. Au Castelet, les chenaux d'écoulement incisés à l'occasion de la lave torrentielle de mai 1986 ont révélé divers détails du dispositif stratigraphique. La coupe la plus éloquente, car découverte sur plus de deux mètres, est celle de la tranchée qui débouche sur la RN 20, à peu près dans la partie médiane du cône.

L'étendue et le volume du cône de déjection du Lagal au Castelet sont sans commune mesure avec son bassin torrentiel. Cette disproportion suppose donc l'engraissement du cône par des apports catastrophiques, ce que suggère pareillement l'épaisseur des épandages. L'examen de la carte topographique à $1 / 25000^{\circ}$ et celui des photos aériennes permettent de mettre en évidence un trait géomorphologique qui donne une idée de la puissance des écoulements torrentiels et de leur capacité morphogénique. Sur la bordure orientale du cône on peut en effet observer une ancienne berge de l'Ariège (fig. 3); mais celle-ci a été repoussée vers le flanc droit de l'auge, où elle

(13) ARMP, Fonds ONF, 3530/13. 

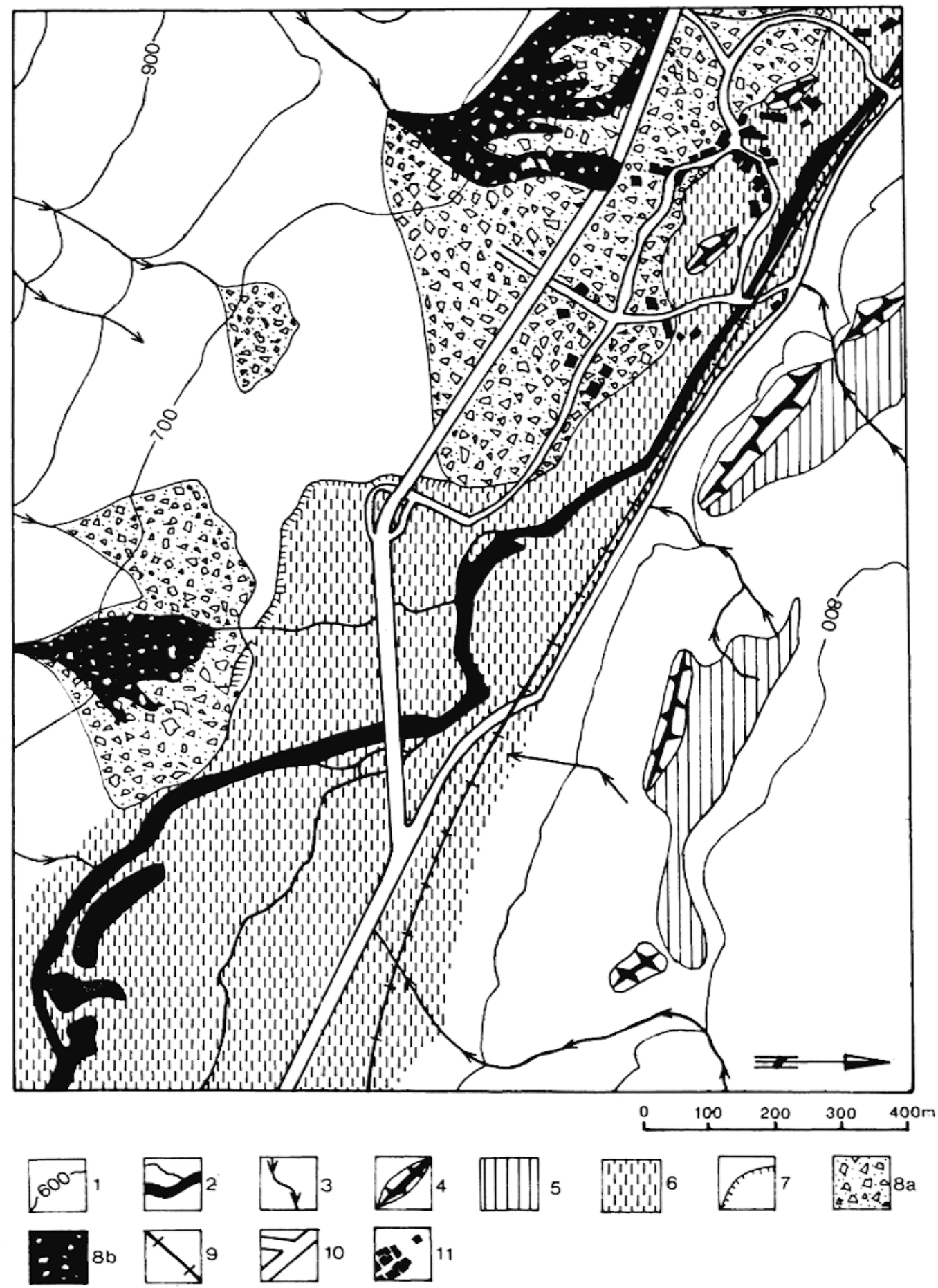

FIG. 3

Le site du Castelet: risques naturels et enjeux socio-6́conomiques.

1. Courbe de niveau. - 2. Rivière et ruisseaux de plaine. -3 . Torrents et chenaux d'érosion des flancs d'auge. - 4. Verrou, îlot rocheux. -5 . Gouttière latérale avec accumulation glaciaire. - 6. Plaine inondable. - 7. Ancienne berge de l'Ariège. $-8 \mathrm{a}$. Cône de déjection. $-8 \mathrm{~b}$. Déjcctions de mai 1986. - 9. Voie ferrée Toulouse-La Tour de Carol. - 10 Réseau routier dont $\mathrm{RN} 20$. -11 . Village 
coule présentement. Les engraissements successifs dus au Lagal et au Rec d'Esquine d'Ase ont donc été assez fréquents et copieux pour pousser à la migration du cours de l'Ariège, celle-ci ne pouvant assurer l'évacuation d'une masse alluviale supérieure à sa capacité.

L'examen du dispositif stratigraphique suggère aujourd'hui ce que furent les remblaiements par le passé. L'incision du cône lors de l'inondation de mai 1986 a mis en évidence trois niveaux bien individualisés : deux niveaux torrentiels entre lesquels s'interstratifie un terme sablo-limoneux brun clair ou grisâtre (fig. 4).

A la base, une séquence caillouteuse, relativement indurée, formée de galets décimétriques emballés dans une matrice de graviers et de granules, dont la partie supérieure porte de nombreuses marques d'oxydation de teinte rouge brunâtre qui témoignent de l'évolution du dépôt dans un milieu aéré.

Suit une strate intermédiaire sablo-limoneuse grisâtre à stratification subhorizontale où des traces d'hydromorphie trahissent une évolution en milieu confiné, réducteur.

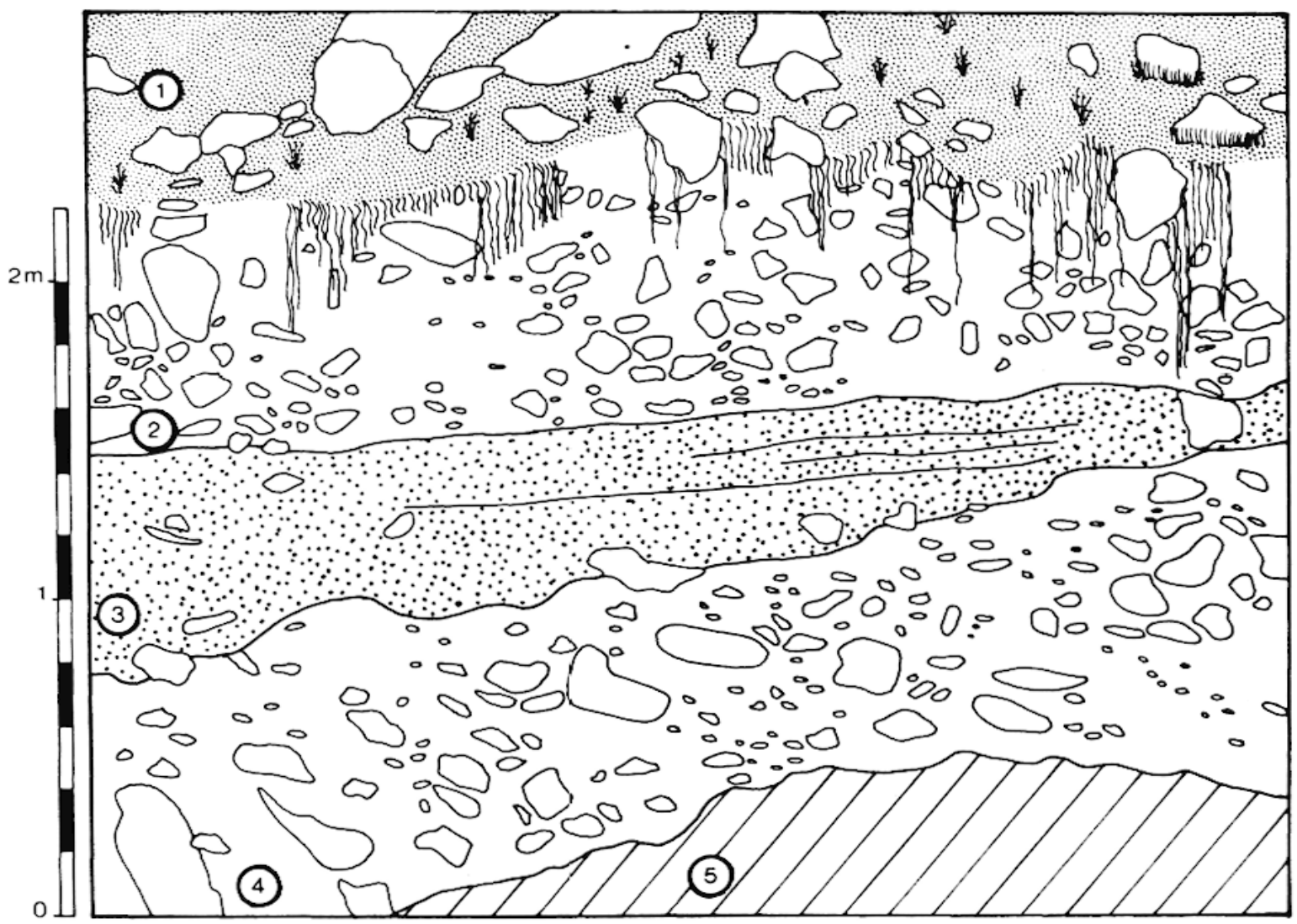

Frg. 4

Coupe du cône du Lagal.

1. Surface actuelle du cône. - 2. Epandage caillouteux grossier indiférencié des des laves de 1875 et 1910 (1881 ?). - 3. Horizon sablo-limoneux avec marques d'hydromorphie. - 4. Niveau caillouteux à matrice grumeleuse comprenant la lave de 1772. - 5. Dépots de lit du chenal et alluvions remaniées en 1986. 
Enfin, le niveau terminal de galets de taille centimétrique à pluricentimétrique emballés dans une matrice grumeleuse ou granuleuse ne présente aucun indice d'oxydation.

Nous pouvons suggérer une interprétation chronologique de cette succession. Le terme supérieur du dépôt torrentiel correspondrait aux laves de 1910 et 1875; mais les coupes dont nous disposons ne laissent percevoir aucune discontinuité qui puisse correspondre à celle des deux événements et il est possible d'admettre que ce niveau comprend aussi des apports attribuables aux épandages beaucoup plus irréguliers de la lave de 1881. Le terme médian du dépôt est d'une autre nature : il révèle une ambiance plus calme, confinée, réductrice, un milieu mal drainé; il a l'apparence de limons de crue saisonnière ou de ruissellement; dans cette partie du dépôt alluvial la présence d'un objet métallique indéterminé suggère une sédimentation d'époque historique. Quant au terme inférieur révélé par cette coupe, sa physionomie est analogue à celle du supérieur : un dépôt torrentiel qui présente çà et là des traces d'oxydation et dans lequel nous proposons de voir le témoin de la lave de septembre 1772.

Dans cette succession de lits caillouteux et de lits sablo-limoneux, qui est observée par ailleurs dans bien d'autres cônes de déjection du val d'Ariège, les premières traduisent-elles un stade paroxystique de l'activité torrentielle et de l'évolution des versants du bassin et les secondes des temps d'évolution plus calme? La question qui se pose est celle du rythme de la torrentialité et de la possibilité de sa lecture dans les coupes établies aux dépens d'un cône de déjection. Les divagations torrentielles au cours d'un même épisode d'engravement sont suffisantes pour introduire des variations latérales très rapide de la physionomie du dépôt alluvial (14). Les progrès de nos analyses reposeront donc moins sur l'examen de la géométrie et de la granulométrie des strates ou des lentilles sédimentaires formant le cône de déjection que sur la recherche des différences d'altération des dépôts in situ et sur l'examen des paléosols. En ce sens la présence de marques d'oxydation dans le terme inférieur de la coupe et leur absence dans le terme supérieur nous paraissent fondamentales; elles nous permettent en effet dans une coupe dont la hauteur est faible, mais que nous pouvons observer sur une longueur assez grande, de repousser la possibilité que les deux séquences à galets soient contemporaines du même épisode torrentiel, représentant deux anciens lits séparés par migration du chenal avec dépôt sur leurs marges de sables plus fins.

La question qui se pose à propos de nos observations relatives à la structure alluviale du cône du Castelet est finalement de savoir si les observations faites sur un seul tracé de coupe, même si cette

(14) G. HéraIl Les cônes de déjection : formes et sédiments, Bull. soc. nat. Elf Aquitaine, 1984, 8, 1, pp. 135-150. 
dernière est longue, peuvent être extrapolées à l'ensemble du cône. Autrement dit, faut-il admettre que de tels cônes courts et assez raides, comme on peut en observer beaucoup dans les Pyrénées peuvent présenter un dispositif alluvial en feuillets ou en bancs successifs superposés plutôt qu'une distribution lenticulaire des alluvions. Les quelques observations faites au Castelet à propos de l'altération de matériaux alluviaux nous inclinent à envisager la première proposition comme vraisemblable.

\section{Deux réalités contemporaines.}

Le site du Castelet peut donc être considéré, tant à partir de l'analyse historique que d'un examen géomorphologique, comme un site à risques latents.

\section{Le cône de déjection du Castelet : un site à risques.}

Le val d'Ariège présente indéniablement des risques sérieux d'une évolution morphogénétique catastrophique. Le nombre de paroxysmes torrentiels enregistrés depuis le XVIII' siècle en témoigne; il faut en replacer la série dans une conjoncture marquée par des phénomènes qui accentuent la sensibilité du milieu aux contraintes climatiques, en particulier ceux qui tiennent à une certaine surexploitation de l'espace.

Le déclenchement de la lave torrentielle du mois de mai 1986 a vérifié la pérennité de ces risques. Un orage violent, suivant un mois pluvicux, à provoquć une crue subite qui, entraînant $1 c$ remaniement des alluvions du chenal torrentiel, a déterminé cette lave. Aucune déchirure d'érosion supplémentaire n'a été ouverte sur les versants, mais deux ravins affluents de rive droite ont été récurés par la même occasion. Les infrastructures du déversoir de la conduite forcée d'EDF, vers $1050 \mathrm{~m}$, ont subi quelques dégâts et sur une bonne moitié du cône de déjection, l'engravement a affecté $80 \%$ de la superficie, les matériaux transportés parcourant et recouvrant cette partie du cône avant de s'engouffrer sous le nouveau pont et de s'épandre plus bas à proximité du village. Cependant à l'issue de la gorge, un bras de la lave a divergé et a emprunté avec une précision remarquable un ancien tracé du ruisseau qui avait été utilisé avant 1875 à peu près selon l'axe du cône. Les alluvions ont alors atteint la RN 20, qui est ici en déblai (fig. 3). Ce furent les seuls dommages notables avec l'engravement du cône, qui ne porte plus aujourd'hui que des landes irrégulièrement pâturées. Mais on imagine aisément ce qu'auraient su être les effets d'événements semblables à ceux de 1772, 1875 ou 1910 : face à des phénomènes de cette importance la sécurité du village, qui n'a jamais été gravement touché, n'est sans doute pas garantie. Cependant, si les menaces qui pèsent sur le village du Castelet et ses habi- 
tants relèvent d'hypothèses maximalistes, la lave torrentielle de mai 1986 signale clairement la fragilité de la RN 20.

Le torrent du Lagal ne pourrait à lui seul entraîner une interruption de la circulation puisque subsiste l'ancien tracé de cette route par la rive droite de l'Ariège. Mais, en cas de crue simultanée de l'Ariège, donc de menace sur cet ancien tracé, la coupure de l'itinéraire serait totale, - avec toutes les conséquences que cela entraînerait en période de trafic intense (tourisme d'été, mouvement vers les stations de sports d'hiver, fréquentation de l'Andorre en fin de semaine). Même si la dernière lave survenue n'a provoqué qu'une interruption négligeable, n'oublions pas que cet épisode torrentiel n'eut pas l'ampleur de certains de ceux qui ont contribué à l'édification du cône de déjection, y compris aux temps historiques.

L'axe de la RN 20 dans le val d'Ariège prend une importance accrue du fait de l'entrée de l'Espagne dans le Marché commun européen et dans la perspective du projet de percement du tunnel du Puymorens; de nouveaux problèmes de sécurité et d'entretien se posent donc. Il faut donc prendre en compte les accès paroxysmaux de l'activité torrentielle dans le bassin du Lagal et ne voir dans ce dernier qu'une illustration de la menace que peuvent représenter les autres tributaires torrentiels de l'Ariège. L'exemple du Lagal montre bien que les "débordements " sont souvent sans commune mesure avec la taille des émissaires. La sous-évaluation du risque est d'autant plus grande que la mémoire collective a oublié non seulement les laves désastreuses de 1772 à 1875 mais paraît minimiser la gravité de celle de 1910 : la commune du Castelet avait même, avant 1988, envisagé de laisser lotir et construire sur le cône de déjection... Le torrent n'est-il pas oublié simplement parce que la société locale est oublieuse?

La vidange survenue de la sorte en mai 1986 est ainsi un bon exemple, à grande échelle, de ce qui peut arriver mais que le temps fait tomber dans l'oubli : n'a-t-on pas reconstruit une partie du village de Verdun, Barry d'en Haut, à l'endroit même où les laves de 1875 , qui firent 81 morts, puis celles de 1897 l'avaient emporté. Mais fautil voir dans l'épisode récent survenu au Castelet, qu'avaient précédé les désastres de novembre 1982 en Haute Ariège, les prémices d'un renouveau de la torrentialité catastrophique qui avait caractérisé le troisième quart du XVIII ${ }^{e}$ siècle et la fin du $\mathrm{XIX}^{*}$ ?

\section{Recrudescence cyclique des processus catastrophiques ?}

Tant la stratigraphie du cône du Castelet que le témoignage des archives nous assurent de la réalité d'un risque permanent et d'un retour cyclique des catastrophes torrentielles.

Les cônes de déjection du val d'Ariège paraissent bien traduire l'alternance de périodes paroxystiques et de périodes de calme relatif. L'ensemble des séquences envisagé pour le Castelet embrasse 
environ deux cent cinquante ans : deux séries d'occurrences torrentielles catastrophiques et trois phases plus calmes : début du XVIII ${ }^{\circ}$ siècle, vers 1780, vers 1850 puis des années 1920 au début de la décennie 1980. La question est de savoir si l'événement de 1986 marquera le début d'une phase d'instabilité.

Or les paroxysmes du Lagal n'apparaissent pas aléatoires : ils sont toujours contemporains de "crises catastrophiques " évidentes dans le reste de l'Ariège aussi bien que dans l'ensemble des Pyrénées (15). Comment interpréter dès lors ces retours cycliques qui pourraient s'inscrire dans une périodicité séculaire ? La distribution préférentielle des catastrophes peut être mise en relation avec l'histoire du climat : la fin du XVIII $\mathrm{X}^{\bullet}$ siècle et celle du $\mathrm{XIX}^{\bullet}$ sont connues comme des phases d'instabilité, d'irrégularité météorologiques, avec des fréquences anormales d'hivers froids, de sécheresses, de tempêtes, etc. Les situations de blocage de dépression, génératrices de grands abats d'eau furent sans doute beaucoup plus nombreuses alors et engendrèrent la plupart des crues catastrophiques des Pyrénées. Les débuts du XVIII ${ }^{e}$ siècle, du $\mathrm{XIX}^{e}$ et du $\mathrm{XX}^{\cdot}$ paraissent en revanche avoir été des phases aux caractères climatiques plus réguliers. Sommes nous, vers la fin de la décennie 1950, arrivés à une nouvelle période d'instabilité, caractérisée par des records de températures ou de précipitations ? Les sécheresses récentes, les derniers hivers froids, les crues en sont-elles des manifestations ? Faut-il, dans cette perspective, s'attendre à de nouveaux paroxysmes dans la montagne pyrénéenne ? Pourrait-on, sur la base des observations rassemblées, les prédire ? Il est clair, en tout cas, que l'on peut, sans verser dans un catastrophisme inutile, affirmer qu'une crise aussi violente et destructrice que celles qui ont marqué le $\mathrm{XVIII}^{\circ}$ siècle et le $\mathrm{XIX}^{\bullet}$ demeure possible. Nul ne peut donc, ni au Castelet ni en d'autres localités exposées du val d'Ariège : Verdun, Luzenac (RN 20 et camping), Albiès (RN 20 et maisons proches), Lassur (RN 20), Bouan, ignorer le risque de tels phénomènes qui menaceraient la sécurité des hommes et l'usage des équipements. Mais il s'agit finalement de décider si l'on tiendra compte, dans les perspectives d'aménagement et dans les budgets correspondants, de phénomènes dangereux, possiblement coûteux, dont la probabilité de l'occurence future paraît grande sans que leur échéance soit encore prévisible.

(15) Des recherches sur ce thème sont en cours dans lc cadre d'un programme PIREN consacré aux Histoires de l'environnement. 


\section{Bibliographie}

J.-M. AntoIne, Méthodologie de la reconstitution des rythmes catastrophiques en val d'Ariège, Mémoire DEA, Institut de géographie, Université de Toulouse-Le Mirail, 1987.

H. Gaussen, Végétation de la moitié orientale des Pyrénées. Sol, climat, végé. tation. Paris, Le Chevallier, 1926.

H.H. LAMB Climate: past, present and future, Londres, Methuen, 1972 et 1977 ( 2 vol.).

J.-P. MÉtailié, Photographie et histoire du paysage : un exemple dans les Pyrénées luchonnaises, RGPSO, 1986, 2.

P. Pédelaborde, Le climat du bassin parisien. Paris, Génin, 1957.

E. Lf. Roy Ladurif, Histoire du climat depuis l'an mil. Paris, Flammarion, 1983. 2 vol. (2' édition).

Revue géographique des Pyrénées et du Sud-Ouest, «Catastrophes », t. 48, fasc. 2, 1986.

Résumé. - Le Val d'Ariège, entre Tarascon et Ax-les-Thermes, a connu une intense activité torrentielle; elle est attestée par les archives au cours de la période XVII-XIX ${ }^{\mathrm{e}}$ siècle et on peut en retrouver les traces dans la stratigraphie des cônes de déjection. La chronologie établie à partir des sources montre une réapparition cyclique des phénomènes torrentiels, qui se concentrent en véritables crises érosives à la fin du XVII', XVIII et XIX siècle. Le torrent du Lagal, au Castelet, est représentatif de cette histoire catastrophique : il a connu une quinzaine de paroxysmes torrentiels en deux siècles et possède un cône important avec une stratigraphie montrant, sur plusieurs mètres d'épaisseur, la succession des phases de calme et de crise. Après 70 ans d'accalmie, le torrent qui avait été un peu oublié a rappelé en 1985 la menace qu'il fait peser, ainsi que plusieurs autres, sur des villages et un axe fréquenté.

SUMMARY. - THE FORGOTTEN TORRENT: THE SPASMODIC NATURE OF THE « TORRENT DU Lagal " IN THE COMMUNe OF PeRles-le-CasteleT, 1771-1986. The Ariège valley between Tarascon and Ax-les-Thermes, has experienced an intense torrential activity: this is proven in the archives from the $17 \mathrm{th}, 18 \mathrm{th}$ and $19 \mathrm{th}$ centuries, and traces of it can be found in the stratification of alluvial cones. The history established from these sources shows a cyclical pattern of torrent phenomena, which are concentrated in genuine erosive crises at the end of the 17th, 18th and 19 th centuries. The Torrent du Lagal, at Perles-le-Castelet reflects this history of hydrological disasters : it has suffered about fifteen torrential crises in two centuries and it has a sizeable cone with a stratigraphy that describes, through a depth of several meters, the alternate phases of calm and crisis. After a lull of 70 years, the torrent, which had been somewhat forgotten, once again, made known its menacing presence in 1985 (as did several other torrents) in a number of villages and along a main road.

Resumen. - El torRente olvidado. Permanencia y Paroxismos de la torRen-

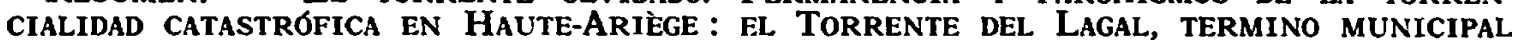
DE Perles-le-Castelet, 1772-1986. El valle del Ariege, entre Tarascon y Ax-lesThermes ha sufrido intensa actividad torrencial. Para el periodo siglos XVII al $\mathrm{XIX}$, los archivos atestan esta actividad cuyas huellas se pueden encontrar en la estratigrafía de los conos de deyección. La cronología establecida partiendo de las fuentes muestra la aparición cíclica de los fenómenos de torrencialidad que representan auténticas crisis de erosión a finales de los siglos XVII, XVIII y XIX. El Torrente del Lagal, en Castelet, es buena muestra de esta historia catastrófica con unos quince paroxismos torrenciales en dos siglos. La estratigrafía de su importante cono muestra, en varios metros de espesor, la sucesión dz las fases de calmé y de crisis. En 1985, olvidado o casi por todo el mundo, y después de 70 años de calma, el torrente ha hecho recordar la amenaza que representa, con otros torrentes, sobre algunos pueblos y un eje de fuerte tráfico.

Mots-Clés. - Pyrénées, Ariège, Val d'Ariège, XVIII ${ }^{e}$ siècle, XIX* siècle, torrentialité, lave torrentielle, crise érosive, cône de déjection, catastrophes. 